Ann. Zootech., I975, 24 (2), 237-242.

\title{
INFLUENCE DU MODE DE PRÉSENTATION DU FOURRAGE SUR LA DIGESTIBILITÉ CHEZ LE PONEY
}

\author{
R. WOLTER, Andrée DURIX et J. C. LETOURNEAU \\ Laboratoive de Recherches de la Chaire d'Alimentation, \\ École nationale vétérinaire de Iyon, \\ 69337 Lyon Cedex 1
}

\section{RÉSUMÉ}

Six poneys, de I $70 \mathrm{~kg}$ en moyenne, placés en cages à métabolisme, reçoivent un même foin de graminée présenté successivement sous forme longuc, broyée ou granulée, et qui a fait parallèlement l'objet d'une étude de la vitesse du transit digestif.

La digestibilité de la matière sèche et de la cellulose est abaissée par le broyage, alors que celle des protéines n'est pas affectée. La granulation, après broyage, tend à diminuer davantage la digestibilité de la matière sèche et à abaisser celle des protéines.

La glycémie ne présente pas de variation marquée en fonction de la présentation du fourrage, alors qu'on observe quelques différences dans les taux sanguins d'acides gras volatils.

\section{INTRODUC'TION}

L'influence de la présentation de la ration sur la vitesse du transit digestif, l'appétit et la digestibilité, a été particulièrement étudiée chez les polygastriques. I,es travaux, beaucoup plus restreints, qui abordent cet aspect de l'alimentation chez les monogastriques, ont surtout été effectués chez le Porc, plus rarement chez les équidés.

Chez les ruminants, la comparaison d'un fourrage utilisé sous forme normale, ou condensée (broyée, puis granulée), a fait l'objet de très nombreuses mises au point, notamment aux États-Unis et en France, par Journet et HodEN (I973), JARRIGE et al., I973). Le bénéfice retiré de la mise en granulés d'un fourrage, quant aux performances d'engraissement, est d'autant plus sensible que le broyage est plus fin et la complémentation concentrée plus limitée (Minson, I962; BEARDSLEY, I964). Il résulte pour une large part de l'augmentation du niveau alimentaire, en rapport 
avec l'accélération du transit ruminal, qui est elle-même liée à l'état divisé de la ration. Le broyage a pour conséquence l'augmentation du rendement de l'énergie métabolisable de l'aliment; on admet actuellement (BREEVER et al., I972) que cette amélioration est due surtout à une diminution de la digestion dans le rumen, consécutive au transit accéléré, au profit de la digestion intestinale, plutôt qu'à une augmentation du rapport acide propionique/acide acétique.

Chez les équidés, HaEnlein et al. (I966), estimant chez le Poney la valeur nutritive d'un même foin broyé, comprimé ou condensé, ne signalent pas de différence de digestibilité entre les trois formes physiques, sauf pour la cellulose brute qui est moins digestible dans le cas du foin condensé. Les quantités ingérées augmentent de $\mathrm{r} 7 \mathrm{p}$. Ioo avec le fourrage comprimé et de $24 \mathrm{p}$. roo avec le fourrage condensé, par rapport à la farine. Hin'rz et Loy (I966) arrivent à des conclusions semblables : la granulation d'une farine n'en modifie pas la digestibilité, sauf celle de la cellulose brute.

Dans le but d'apporter quelques précisions sur ce sujet, nous avons examiné, chez six poneys, les répercussions de la présentation d'un même foin, distribué sous forme longue, broyée ou condensée, sur la digestibilité de différents composants de la ration : matière sèche, cellulose, protéines; parallèlement, nous avons mesuré les taux plasmatiques de glucose et d'acides gras volatils. L'influence du mode de présentation sur la vitesse du transit digestif a déjà été rapportée (WoLTER et al., I974).

L'essai a été conduit en alimentation restreinte, correspondant approximativement à une ration d'entretien, puisque, chez le Cheval, la fraction condensée de la ration est le plus souvent limitée car on recherche rarement les performances zootechniques maximales. Ainsi, nous pouvons juger de l'influence propre de la ration, indépendamment des conséquences de la présentation de celle-ci sur le niveau de consommation volontaire, si important chez les ruminants.

\section{I. - CONDITIONS EXPÉRIMENTALES}

Six poneys mâles, dont le poids moyen passe de $\mathrm{I} 64 \mathrm{~kg}$ au début de l'expérimentation à $174 \mathrm{~kg}$ à la fin, sont placés en cages à métabolisme individuclles.

L'aliment est constitué par un foin de graminées (contenant très peu de luzerne), présenté sous trois formes : longue, broyée et condensée. Ne disposant pas de moyens de préparation de nos aliments, nous avons utilisé un lot de fourrage du commerce. Le broyage des deux tiers du lot a été réalisé dans un broyeur muni d'une grille $5 \% /$ roo $\mathrm{mm}$ et une partie de cette farine a été ensuite granulée dans une presse Russell (filière de $9,5 \mathrm{~mm}$ de diamètre). L'hétérogénéité du foin, ainsi qu'une ségrégation des constituants physiques intervenue au cours de la préparation, sont sans doute la cause des différences de composition chimique entre les trois présentations du fourrage (tabl. I).

Pour chaque forme d'aliment, les animaux sont soumis à une période d'adaptation de quinze jours ; puis, pendant une semaine, ils sont rationnés à $4 \mathrm{~kg}$, la distribution du fourrage se faisant en deux fois, à 8 heures et 17 heures. La période expérimentale s'étend ensuite sur huit jours consécutifs. Les fèces sont récoltées deux fois par jour, et homogénéisées; un échantillon de ıo p. i oo du poids frais est prélevé en vue de la détermination de la matière sèche, par séchage à l'étuve à $100^{\circ} \mathrm{C}$. Le mélange des fèces séchées d'une même période est alors broyé afin de pouvoir évaluer ses teneurs en matières azotées totales $(\mathrm{N} \mathrm{Kjeldhal} \times 6,25)$ et en cellulose brute de Weende.

Lcs taux de glucose sanguin et d'acides gras volatils (acides acétique, propionique, butyrique) ont été déterminés à partir d'échantillons de sang jugulaire prélevé à I 4 heures, pendant trois jours consécutifs, durant la semaine qui précède les mesures de digestibilité. La glycémie est 
TABIEAU I

Composition du foin sous les trois présentations différentes (en p. Ioo)

\begin{tabular}{c|c|c|c}
\hline & Matière sèche & $\begin{array}{c}\text { Cellulose brute } \\
\text { de Weende }\end{array}$ & $\begin{array}{c}\text { Matières azotées } \\
\text { totales }(\mathrm{N} \times \mathbf{6 , 2 5})\end{array}$ \\
\hline Foin long & 90,91 & 31,19 & 5,46 \\
\hline Foin broyé & 91,19 & 34,96 & 7,06 \\
\hline Foin granulé & 92,66 & 33,70 & 6,79 \\
\hline
\end{tabular}

estimée par la méthode colorimétrique de Folin-Wu (adaptée au photocolorimètre LumetronÉtablissements Photonolt Corporation), à partir de sang recueilli sur fluorure de sodium. Les acides gras volatils ont été dosés par chromatographie en phase gazeuse (BAUMGARDT, I964), à partir de sang total recueilli sur héparine et conservé au congélateur.

\section{II. - RÉSULTATS ETT DISCUSSION}

Les coefficients de digestibilité apparente de la matière sèche et de la cellulose brute sont nettement plus élevés pour le foin long que pour les formes broyée et condensée (tabl, 2).

\section{TABLEAU 2}

Coefficients d'utilisation digestive et taux plasmatiques de glucose et d'acides gras volatils

\begin{tabular}{|c|c|c|c|c|c|c|c|}
\hline & & \multicolumn{2}{|c|}{ Foin long } & \multicolumn{2}{|c|}{ Foin broyé } & \multicolumn{2}{|c|}{ Foin condensé } \\
\hline & & Moyenne & $\begin{array}{l}\text { Ecart- } \\
\text { type }\end{array}$ & Moyenne & $\begin{array}{l}\text { Ecart- } \\
\text { type }\end{array}$ & Moyenne & $\begin{array}{l}\text { Écart- } \\
\text { type }\end{array}$ \\
\hline \multirow{3}{*}{$\begin{array}{l}\text { CUID } \\
\text { p. } 100\end{array}$} & Matière sèche & $57,81 A *$ & $\pm \quad 1,7$ & $48,9 \div 8$ & $\therefore \quad \overline{3}, \dot{3}$ & $43,655^{B}$ & $\pm \quad 4,9$ \\
\hline & Matières cellulosique: & $+1,31 a *$ & $\pm 5,0$ & $31,49^{b}$ & $\therefore \quad 8,9$ & 33,71 & $\pm 10,1$ \\
\hline & Matières protéiques & 50,23 & $\pm \quad 5,2$ & $55,1^{\prime}+$ & $\pm 4, N$ & 49,41 & $\pm 8,5$ \\
\hline \multicolumn{2}{|c|}{ Glucose plasmatique (g/1) } & $0,90 a$ & $\pm 0,12$ & $1,09^{b}$ & $\doteq 0,05$ & $1,016^{b}$ & $\pm 0,06$ \\
\hline \multirow{4}{*}{$\begin{array}{l}\text { Acides } \\
\text { gras } \\
\text { volatils }\end{array}$} & $\begin{array}{l}\text { Ac. acétique }(\mathrm{mg} / \mathrm{l}) \\
\text { (p. lo0 du total des } \mathrm{A}(\mathrm{iN})\end{array}$ & $\begin{array}{l}87,34 A \\
85,7\end{array}$ & $\pm 18,9$ & $\begin{array}{c}135,69 B \\
91,9\end{array}$ & $=1: 3$ & $\begin{array}{l}85,62 A \\
88,6\end{array}$ & $\pm 31,9$ \\
\hline & $\begin{array}{l}\text { Ac. propionique (mg/l) } \\
\text { (p lou du total des A(i) })\end{array}$ & $\begin{array}{l}13,05 \\
12, x\end{array}$ & $\pm \quad 3,5$ & $\begin{array}{c}10,6 t \\
7,2\end{array}$ & $\pm \quad 2,17$ & $\begin{array}{l}10,05 \\
10,5\end{array}$ & $\pm 0,99$ \\
\hline & $\begin{array}{l}\text { Ac. butyrique }(\mathrm{mg} / \mathrm{l}) \\
\text { (p. Ino du total des } A(\mathrm{iV})\end{array}$ & $\begin{array}{l}1,45 \\
1,42\end{array}$ & $\pm \quad 0,96$ & $\begin{array}{l}1,25 \\
0,81\end{array}$ & $\doteqdot 0,51$ & $\begin{array}{l}0,81 \\
0,81\end{array}$ & $\pm 0,20$ \\
\hline & Acides totaux $(\mathrm{m} N / 1)$ & 1,63 & & 2,11 & & 1,63 & \\
\hline
\end{tabular}

* Les valeurs affectées de lettres différentes en indice diffèrent significativement : majuscule $\mathrm{P}<0,001$; minuscule $\mathrm{P}<0,05$. 
Ces résultats sont à interpréter en fonction des vitesses de transit digestif obtenues avec les mêmes aliments (WOLTER et al., I974). On constate en effet que les coefficients de digestibilité de la cellulose brute se classent dans l'ordre du temps de séjour moyen des fourrages dans le tube digestif : pour les formes longue, broyée et condensée, les coefficients de digestibilité sont, respectivement, ${ }^{\prime}$ de $4 \mathrm{I}, 3,3 \mathrm{I}, 5$ et 33,7 p. Ioo, et les temps de séjour moyen, de $36 \mathrm{~h} 50$, de $25 \mathrm{~h} 5^{2}$ et $3 \mathrm{I} \mathrm{h} \mathrm{I3.} \mathrm{Il} \mathrm{apparait}$ donc une relation négative entre la vitesse du transit digestif de la ration et le coefficient d'utilisation digestive de la fraction cellulosique. Un temps de séjour plus long favorise sans doute l'attaque des éléments cellulosiques par les bactéries du gros intestin. L'augmentation de l'aptitude à la cellulolyse résultant de l'état de division des foins sous forme broyée et condensée, ne suffirait pas alors”à compenser la réduction du temps de séjour. Toutefois, les taux de cellulose légèrement supérieurs des foins broyé et condensé peuvent aussi rendre compte de la diminution de la digestibilité.

Les différences entre les digestibilités sont très atténuées dans le cas des matières azotées. Le coefficient d'utilisation digestive apparent est légèrement plus élevé avec le foin broyé (55 p. IOO) qu'avec le foin long (53,2 p. IOo) ou la forme condensée. Cependant, les différences liées au traitement ne sont pas significatives. Alors que la cellulose est soumise à une action bactérienne tardive et lente se produisant dans le cæcum et le colon, la digestion des matières azotées est due à une attaque enzymatique précoce et rapide se déroulant en majeure partie dans l'intestin grêle, comme cela a été vérifié chez l'Ane (Wol.TER et VÉLANDIA, I97o) et chez le Poney (REITNour et al., I970). De ce fait, la digestibilité des protéines est moins sensible à la vitesse du transit digestif.

Ces résultats confirment que le comportement digestif du Poney se rapproche de celui d'un monogastrique.

Les taux plasmatiques de glucose sont significativement différents avec le foin long par comparaison avec les autres présentations du fourrage. Ces résultats sont quelque peu en désaccord avec ceux de Hintz et al. (I97I), qui ne constatent pas de variation de la glycémie avec des régimes comportant différentes proportions de fourrage et de grains; ces auteurs admettent que les propriétés glucogéniques des rations sont compensées par une bonne régulation hormonale.

En ce qui concerne les acides gras volatils sanguins, la présentation physique de l'aliment n'entraine pas de différence significative des taux d'acide propionique et butyrique, ce qui pourrait expliquer les très faibles variations de la glycémie. En effet, Argenzio et HinTz (I970) ont montré que, parmi les acides gras volatils, seul le propionate retentit significativement sur le taux de glucose sanguin chez le Poney à jeun, alors qu'il n'a aucun effet sur l'animal alimenté. Cependant ces résultats diffèrent un peu de ceux de Kennedy et al. (I966) qui ne signalent pas d'acide propionique dans le sang périphérique chez le Poney. Par contre nous obtenons un taux d'acide acétique significativement plus élevé $(\mathrm{P}<0, \mathrm{OI})$ avec l'aliment broyé qu'avec les formes longue ou condensée.

Ces résultats sont en accord avec ceux de JEAN-BLAIN (I973) : chez le Cheval, le taux d'acide acétique diminue lorsque le foin est complémenté avec de l'avoine, et surtout lorsqu'il est remplacé par un aliment complet; le taux d'acide propionique n'est significativement différent qu'avec l'aliment complet, alors qu'il n'y a aucune évolution du taux d'acide butyrique avec la nature du régime. 
Dans les conditions d'une alimentation rationnée, le broyage, puis la granulation d'un fourrage entraînent, chez le Poney, une diminution de la digestibilité de la matière sèche et des matières cellulosiques, mais affectent peu l'utilisation des matières azotées. Cela est conforme aux variations du transit digestif chez les équidés en fonction du mode de présentation de l'aliment. La glycémie ne varie pas, alors que parmi les acides gras volatils, seul le taux d'acide acétique croît avec le régime foin broyé.

Toutefois, ces résultats ne donnent qu'une idée globale des processus digestifs chez le Poney. Ils demandent à être étendus à différents régimes, en particulier à des aliments complets sous différentes présentations. Par ailleurs, des prélèvements du contenu du tube digestif effectués à plusieurs niveaux, à l'aide de fistules permanentes, donneraient des indications précieuses sur le site et les produits de digestion de la ration en fonction de sa composition et de son conditionnement.

Reçu pour publication en janvier 1975.

\section{SUMMARY}

\section{FORAGE, DIGESTIBILITY IN THE PONEY AS AFFECTED BY THE PHYSICAL, FORM OF THE RATION}

Six poneys, weighing on an average $170 \mathrm{~kg}$, were placed in metabolism crates and received the same grass hay offered either long (normal), ground or pelleted. The digestibility was studied in relation to the rate of passage of the forage through the digestive tract.

Digestibility of dry matter and crude fiber was reduced by the grinding of the forage, whereas protein was not affected. Pelleting, after grinding, tended to decrease even more the digestibility of the dry matter and reduced that of the protein.

The blood glucose level did not show any marked variation in relation to the physical form of the forage, whereas some differences were observed as regards the blood levels of volatile fatty acids.

\section{RÉFÉRE,NCES BIBLIOGRAPHIQUES}

Argenzio R. A., Hintz H. F., I970, Glucose tolerance and effect of volatile fatty acids on plasma glucose concentrations in ponies. J. Anim. Sci, 30, 5I4-5 I8.

Baumgardt B. R., Ig64. Practical observations on the quantitative analysis of free volatil fatty acids in aquous solutions by gaz liquid chromatography. Departemental bulletin 1, Department of dairy science, Lniversity of Visconsin, Madison.

BEARDSLEy D. M., I964. Symposium on forage utilization; nutritive value of forage as affected by physical form. Part II : Beef cattle and sheep studies. J. Anim. Sci., 23, 239-245.

Breever D. E., Coelho da Silva J. F., Prescott J. H., Armstrong D. G., ig72. The effect in sheep of physical form and stage of growth on the sites of digestion of a dried grass. $B r . J$. Nutr., $28,347-356$.

Haenleiv G. F. W., Holdren R. D., Yoon Y. M., ig66. Comparative response of horses and sheep to different physical forms of alfalfa hay. J. Anim. Sci., 25, 740-743.

Hin't H. F., Loy R. G., I966. Effects of pelleting on the nutritive value of horse rations. J. Anim. Sci., 25, I059-1062.

Hintz H. F., Argenzio R. A., Schryver H. F, i97r. Digestion coefficients, blood glucose levels and molar percentage of volatile acids in intestinal fluid ponies fed varying forage-grain ratios. J. Anim. Sci, 33, $992-995$.

Hintz H. F., Hogue D. E., Walker E. F., Lowe J. E., Schryver H. F., ig7i. Apparent digestion in various segments of the digestive tract of ponies fed diets with varying roughage-grain ratios. $J$. Anim. Sci., 32, 245. 
Jarrige R., Demarquilly C., Journet M., Beranger C., 1973. The nutritive value of processed deshydrated forages with special reference to the influence of physical form and particle size. First International Green Crop Drying Congress Oxford England, 9-13.

JEAn-Blain $\quad$ C., I973. Variation de la teneur en acides gras volatils du sang périphérique chez le Cheval, en fonction du régime alimentaire et de l'activité musculaire. Ann. Biol. anim. Bioch. Biophys., 13, $763-764$.

Journet M., Hoden A., r973. Utilisation des luzernes et des graminées déshydratées comme unique fourrage de la ration des vaches laitières et études de leur digestion dans le rumen. Ann. Zootech., 22, 37-54.

Kennedy L. G., Little C. O., Mitchell G. E., Stowe H. D., Rodney J. R., ig66. Glucose and volatile fatty acids in equine caecal fluid and blood. Abstracts. 25, 892.

Minson D. J., I962. The effect of grinding, pelleting and wafering on the feeding value of roughages : a review. Contrib. no $8 \$$ Anim., Res Inst. Canada. Department Agr., Ottawa, Ontario.

Reitnour C., Baker J. P., Mitcheld G. E., Little C. O., Kratzer D. D., i97o. Aminoacids in equine caecal contents, caecal bacteria and serum. $J$. Nutr., 100, 349-354.

Wolter R., Vélandia J., ig7o. Digestion des fourrages chez l'Ane. Rec. Med. Vet., tome CXLVI, I 4 I-I 52 .

Wolter R., Durix A., Letourneau J. C., I974. Influence du mode de présentation du fourrage sur la vitesse du transit digestif chez le Poney. Ann. Zootch., 23, 293-300. 